\title{
A METHOD FOR SOLVING THE PERIODIC PROBLEM FOR THE $K d V$ EQUATION AND ITS GENERALIZATIONS
}

\section{S. P. NOVIKOV}

1. In 1974, the author, together with Dubrovin, Matveev, and Its, undertook a study of the ideas of Gardner, Greene, Kruskal, and Miura (1967), and investigated an interesting class of "finite-gap" or "multisoliton" solutions of the $K d V$ equation. This involved a considerable extension of the then available theory of the one-dimensional Schrödinger operator (a reasonably detailed exposition may be found in [2]). Let $L=-\left(d^{2} / d x^{2}\right)+u$ be the Sturm-Liouville (Schrödinger) operator with real periodic potential. The Bloch eigenfunctions $\psi_{ \pm}\left(x, x_{0}, E\right)$ are those solutions of $L \psi=E \psi$ which satisfy: $\psi=1$ for $x=x_{0}$, and $\psi_{ \pm}\left(x+T, x_{0}, E\right)=e^{ \pm i p(E) T} \psi_{ \pm}\left(x, x_{0}, E\right)$ (i.e., they are eigenvectors of the translation or monodromy matrix, $\hat{T} \psi(x)=\psi(x, T)) . \psi_{ \pm}\left(x, x_{0}, E\right)$ are defined for all complex $E$, and are branches of a single function $\psi$ meromorphic on the Riemann surface $R$ which is a two-sheeted covering of the $E$-plane with branch points $E_{i}$ at the endpoints of the gaps. Each forbidden band of the spectrum contains a zero of $\psi\left(x, x_{0}, E\right)$ on one sheet of $R$, and a pole on the other. As $E \rightarrow \infty$, one has $\psi \sim \exp \left[ \pm i E^{1 / 2}\left(x-x_{0}\right)\right]$. The zeroes of $\psi$ may be represented in the form $\left(\gamma_{j}(x), \pm\right)$, and the poles as $\left(\gamma_{j}\left(x_{0}\right), \pm\right)$, where + and - identify the sheets. One may think of $\gamma_{j}$ as an eigenvalue of a supplementary Sturm-Liouville problem. The potential $u(x)$ itself may be represented in the form $u(x)=-2 \Sigma_{j} \gamma_{j}(x)+$ const. If the number of gaps is finite, the Riemann surface $R$ is algebraic and has genus $n$ (= the number of gaps).

These are the analytic properties of $\psi$ for real, smooth, periodic potentials. It should be noted that the function $\psi_{ \pm} \equiv-i\left(d \ln \psi_{ \pm} / d x\right)$ is also periodic with period $T$, and (for the case of a finite-gap potential) is an algebraic function on $R$ (even at infinity). Its real part has the form

$$
\chi_{R}=(R(E))^{1 / 2} \prod_{j=1}^{n}\left(E-\gamma_{j}(x)\right)^{-1},
$$

where $R(E)=\Pi_{i=1}^{2 n+1}\left(E-E_{i}\right)$; this is just the Wronskian

$$
\chi_{R}=\frac{1}{2 i}\left(\psi_{+}^{\prime} \psi_{-}-\psi_{-}^{\prime} \psi_{+}\right)
$$


2. We now introduce a class of complex, meromorphic potentials $u(x)$ defined in a strip near the real axis, and almost periodic there. We say that $u(x)$ has "good analytic properties," if there exist eigenfunctions $\psi_{ \pm}\left(x, x_{0}, E\right)$ with these properties.

(i) $L \psi=E \psi$;

(ii) $\psi=1$ for $x=x_{0}$;

(iii) the logarithmic derivative $\chi=-i(d \ln \psi / d x)$ has the same period group as $u(x)$;

(iv) $\psi_{ \pm}$is meromorphic on some two-sheeted Riemann surface $R$ over the $E$-plane, $\psi_{ \pm} \rightarrow \exp \left[ \pm i E^{1 / 2}\left(x-x_{0}\right)\right]$ as $E \rightarrow \infty$, where $\psi_{+}$and $\psi_{-}$go into each other by an interchange of sheets. The branch points of the Riemann surface will be called "endpoints of gaps," and the Riemann surface, the "spectrum."

If the Riemann surface $R$ is algebraic (has finitely many branch points), we call the potential $u(x)$ "finite-gap." In this case, the function $\psi_{ \pm}$has $n$ poles of the form $Q_{j}\left(x_{0}\right)$, and $n$ zeroes of the form $Q_{j}(x)$, where $j=1, \cdots, n$, and the $Q_{j}$ are points of $R$ which may be represented in the form $Q_{j}=\left(\gamma_{j}, \pm\right)$; the potential itself will have the form

$$
u(x)=-2 \sum \gamma_{j}(x)+\sum E_{j},
$$

$E_{j}$ being the branch points.

These facts, together with the ideas of Akhiezer's 1961 paper [13], suffice for the expression of the potential in terms of $\theta$ functions. The most elegant formula is [12]

$$
u(x)=-2 \frac{d^{2}}{d x^{2}} \ln \theta\left(x U_{1}+\eta_{1}^{\circ}, \cdots, x U_{n}+\eta_{n}^{\circ}\right), \quad U_{j}=\text { const. }
$$

where $\theta\left(\eta_{1}, \cdots, \eta_{n}\right)$ is the Riemann theta function associated with $R$ (the standard literature on theta functions leads to a less useful result).

3. That this class of potentials is a natural one to study can be seen as follows. Consider the Wronskian $\chi_{R}=(1 / 2 i)\left(\psi_{+}^{\prime} \psi_{-}-\psi_{-}{ }^{\prime} \psi_{+}\right)$. We have $-i \chi^{\prime}+\chi^{2}+u-E=0, \chi=\chi_{R}+(i / 2)\left(\ln \chi_{R}\right)^{\prime}$. As $E \rightarrow \infty$,

$$
\chi \sim E^{1 / 2}+\sum_{n \geqq 1} \frac{\chi_{n}\left(u, u^{\prime}, \cdots\right)}{\left(2 E^{1 / 2}\right)^{n}},
$$

where the $\chi_{n}$ are polynomials, and $\chi_{2 m}$ is a perfect derivative. The quantities

$$
I_{n}=\frac{1}{T} \int \chi_{2 n+3} d x
$$


(or the mean values $I_{n}=\bar{\chi}_{2 n+3}$ in the a.p. case) give rise to the "higher $K d V$ equations"

$$
\dot{u}=\frac{\partial}{\partial x}\left(\frac{\delta I_{n}}{\delta u(x)}+c_{1} \frac{\delta I_{n-1}}{\delta u(x)}+\cdots+c_{n} \frac{\delta I_{0}}{\delta u(x)}\right),
$$

which are mutually commuting and admit a "Lax representation" (1968),

$$
\dot{L}=\left[L, A_{n}+c_{1} A_{n-1}+\cdots+c_{n} A_{0}\right]
$$

The following important fact was discovered: stationary periodic solutions of these equations (i.e., $\dot{u}=0$ ) are finite-gap potentials. The Borg uniqueness theorem implies that all smooth, periodic, finite-gap potentials are obtained in this fashion. (This was shown by several authors.) However, not all solutions of these equations are periodic. The stationary equation turns out to be a completely integrable Hamiltonian system in $x$ with $n$ degrees of freedom; all the constants $c_{j}$ and the commuting integrals can be expressed in terms of the endpoints of the gaps. Therefore, the general solution of the stationary equation (expressed via Riemann theta functions) turns out to be a finite-gap, meromorphic conditionally periodic complex potential, for which the direct and inverse problems are automatically solved (for arbitrary complex "gap boundaries" or branch points).

Of physical interest are the real, bounded (in $x$ ), and conditionally periodic (in $x$ and $t$ ) solutions of the $K d V$ equation $u=6 u u^{\prime}-u^{\prime \prime \prime}$. The stationary equation

$$
\frac{\delta I_{n}}{\delta u(x)}+c_{1} \frac{\delta I_{n-1}}{\delta u(x)}+\cdots+c_{n} \frac{\delta I_{n}}{\delta u(x)}=c_{-1}
$$

has $n$ commuting integrals $J_{1}, \cdots, J_{n}$; if one knows these, one knows also the spectrum, i.e., the Riemann surface $R$. On the general level surfaces $J_{1}=$ const, $\cdots, J_{n}=$ const, the natural variables are the zeroes of the function $\psi_{ \pm}\left(x, x_{0}, E\right), Q_{j}=\left(\gamma_{j}(x), \pm\right)$, and the equation in $x$ takes on the form

$$
\gamma_{\alpha}^{\prime}=\frac{2 i\left(R\left(\gamma_{\alpha}\right)\right)^{1 / 2}}{\prod_{\beta \neq \alpha}\left(\gamma_{\alpha}-\gamma_{\beta}\right)}
$$

The time-evolution of the potential in the $K d V$ is

$$
\dot{\gamma}_{\alpha}=\frac{8 i\left(\sum_{\beta \neq \alpha} \gamma_{\beta}-\frac{1}{2} \sum E_{i}\right)}{\prod_{\beta \neq \alpha}\left(\gamma_{\alpha}-\gamma_{\beta}\right)} .
$$


All these equations can be linearized by the standard Abel transformation

$$
\eta_{k}=\sum_{j=1}^{n} \int_{\left(\gamma _ { j } \left(x_{0), \pm)}\right.\right.}^{\left(\gamma_{j}(x) \pm\right)} \omega_{k}, \quad k=1, \cdots, n
$$

(the integral extending over a path from the poles to the zeroes on $R$ ), where

$$
\omega_{k}=\sum_{q=1}^{n} c_{k q} E^{q-1} \frac{d E}{(R(E))^{1 / 2}}
$$

is some basis for the differentials of the first kind (i.e., without poles) on $R$. The variables $\eta_{k}$, defined on a complex Jacobi variety, are "angles," with $\dot{\eta}_{k}=$ const by virtue of the higher $K d V$ 's (one can also compute the conjugate "action" variables, as Flaschka and McLaughlin showed in a recent preprint).

All finite-gap potentials are conditionally periodic, meromorphic, functions of complex $x$ (in the whole $x$-plane) with $2 n$ periods $\left(T_{1}, \cdots\right.$, $T_{n}, T_{1}{ }^{\prime}, \cdots, T_{n}{ }^{\prime}$ ) defined entirely by the gap boundaries (alternatively, by the surface $R$ ).

4. It is interesting to observe that the imaginary periods $\left(U_{1}, \cdots, U_{n}\right)$ of the potential can be defined through integrals (over cycles of $R$ ) of the differential $d p(E)$. Here $p(E)$ is the quasi-momentum, $\psi_{ \pm}(x+T)=e^{ \pm i p(E) T} \psi_{ \pm}(x)$, and $d p(E)$ has a second-order pole at the branch point at infinity. In the conditionally periodic case this does indeed define the differential of the quasi-momentum, $d p(E)$. The variational derivative $\delta p / \delta u(x)$ has the form (see [11]):

$$
\frac{\delta p}{\delta u(x)}=-\frac{1}{2 \chi_{R}}, \text { where } p(E)=\frac{1}{T} \int_{x_{0}}^{x_{0}+T} \chi_{R} d x
$$

(in the periodic case; $p(E)$ is the mean value $\bar{\chi}_{R}$ in the a.p. case). Since the Wronskian has the form

$$
\chi_{R}=\frac{(R(E))^{1 / 2}}{\prod_{j}\left(E-\gamma_{j}(x)\right)},
$$

these identities show that any complex meromorphic finite-gap potential satisfies one of the stationary higher $K d V$ equations obtained by expanding $\chi_{R}{ }^{-1}$ in powers of $1 / E^{1 / 2}, E \rightarrow \infty$. The form of the Wronskian $\chi_{R}$, and of its expansion for $E \rightarrow \infty$, also lead to expressions for all symmetric polynomials in $\gamma_{1}, \cdots, \gamma_{n}$ through $\left(u, u^{\prime} \cdots\right)$ and the gap boundaries (that is, through the phase-space variables of the higher stationary $K d V$ equations, through which the potential $u(x)$ is defined). 
All the "frequencies" $\dot{\eta}_{k}$ can, by virtue of the higher $K d V$ 's, be computed in terms of the periods of differentials on $R$ which have a pole at infinity, normed by the choice of some basis for the differentials of the first kind on $R$ (see [2], [4]).

5. Separate interest attaches to the only recently analyzed question of the connection between the various procedures for constructing integrals of the stationary higher $K d V$ equations,

$$
\frac{\delta I_{n}}{\delta u(x)}+\sum c_{i} \frac{\delta I_{n-i}}{\delta u(x)}-c_{-1}=0 .
$$

Since $\dot{L}=\left[L, A_{n}+\sum_{i=1}^{n} c_{i} A_{n-i}\right]$, the operator $A=A_{n}+\sum c_{i} A_{n-i}$ acts on the eigenspace $H_{E}$ of solutions of $L \phi=E \phi$. The determinant of $\left.A\right|_{H_{E}} \equiv \Lambda(E)$ is denoted by $-R(E)$. The characteristic polynomial has the form $\operatorname{det}(\lambda-\Lambda(E))=\lambda^{2}-R(E)$. As was shown in [1], the zeroes of $R(E)=0$ determine the boundaries of the gaps for the potential $u(x)$, and the Riemann surface $R$ is determined by the polynomial $\operatorname{det}(\lambda-\Lambda(E))=\lambda^{2}-R(E)$. The coefficients of the polynomial

$$
R(E)=E^{2 n+1}+a_{1} E^{2 n}+\cdots+a_{n+1} E^{n}+\cdots+a_{2 n+1}
$$

are easily expressed through $u, u^{\prime}, \cdots, u^{(2 n-1)}$, and are commuting integrals of the higher stationary $K d V$ equations (qua Hamiltonian system in $x$ ). In fact, the coefficients $a_{1}, \cdots, a_{n+1}$ are expressible in terms of $c_{-1}, \cdots, c_{n}$, and the integrals are $a_{n+2}=J_{1}, a_{n+3}=J_{2}, \cdots, a_{2 n+1}=J_{n}$. The stationary equations have the form

$$
\frac{d \Lambda}{d x}=[\Lambda, Q]
$$

where $\Lambda$ and $Q$ are second-order matrices depending polynomially on $E$. The translation (or monodromy) matrix $\hat{T}$ (for which $\hat{T} \phi(x)=\phi(x+T))$ acts on the eigenspace $H_{E}(L \phi=E \phi)$. It depends on $E, t$, and $x$, and satisfies the equations

$$
\frac{\partial \hat{T}}{\partial t}=[\Lambda, \hat{T}], \frac{\partial \hat{T}}{\partial x}=[Q, \hat{T}]
$$

(the $x$-dependence arises from the choice of a basis in $H_{E}$ ). The integrability condition

$$
\frac{\partial \Lambda}{\partial x}-\frac{\partial Q}{\partial t}=[\Lambda, Q]
$$

leads to equations for $\Lambda$ and $Q$ that hold in all cases (including the conditionally periodic). In the stationary problem, where

$$
\frac{\partial \Lambda}{\partial t}=\frac{\partial Q}{\partial t}=\frac{\partial \hat{T}}{\partial t}=0,
$$


we have the equations

$$
\frac{\partial \Lambda}{\partial x}=[\Lambda, Q] \text { and }[\hat{T}, \Lambda]=0 .
$$

Since $\hat{T}_{\psi}=e^{ \pm i p T} \psi$, the Bloch eigenfunction $\psi$ is also an eigenvector of $\Lambda(E)$ and is defined on the Riemann surface $R$ of $\operatorname{det}(\lambda-\Lambda(E))=0$.

Lax [5] and Gel'fand and Dikir [6] gave a different construction for the integrals of the stationary problem. Since the Poisson-Gardner bracket on functions of $u(x)$ has the form

$$
\left[I, J=\int \frac{\delta I}{\delta u} \frac{d}{d x} \frac{\delta J}{\delta u} d x,\right.
$$

and since the integrals $I_{n}, I_{m}$ commute (Zaharov, Faddeev; Gardner (1971)), one has

$$
\frac{\delta I_{k}}{\delta u(x)} \frac{d}{d x} \frac{\delta J}{\delta u(x)}=\frac{d Q_{k}}{d x},
$$

where $J=I_{n}+\sum c_{i} I_{n-i}+c_{-1} I_{-1}, I_{-1} \equiv-\int u d x$. This, however, implies that $Q_{k}$ is an integral of the stationary problem

$$
\frac{d}{d x} \frac{\delta J}{\delta u(x)}=0 \text {. }
$$

The author and O. I. Bogojavlenskii [7] established the following simple and general proposition: a function $Q$ is a finite-dimensional Hamiltonian for a flow

$$
\dot{u}=\frac{d}{d x} \frac{\delta I}{\delta u(x)}
$$

restricted to the set of fixed points of a second flow,

$$
\frac{d}{d x} \frac{\delta J}{\delta u(x)}=0
$$

in the finite-dimensional phase-space of this system as an equation in $x$, if

$$
[I, J]=0 \text { and } \frac{d Q}{d x}=\frac{\delta I}{\delta u} \frac{d}{d x} \frac{\delta I}{\delta u} .
$$

(Of course, this fact trivially implies the commutativity of the higher $K d V$ integrals.) Bogojavlenskii [8] obtained formulas for the polynomial $R(E)$ in terms of integrals $Q_{k}$, and vice versa. In particular, $\left(J_{1}, \cdots, J_{n}\right)$ depend linearly on $Q_{0}, \cdots, Q_{n-1}$ according to the formula 


$$
J_{i}=2^{2(n-i)+3}\left(\sum_{k+\ell=n+i+1}^{n+1} c_{k} c_{\ell}-4 \sum_{0<\ell \leqslant i} Q_{\ell-1} c_{i-\ell}\right)
$$

where $1 \leqq i \leqq n$. For the Hamiltonians of the higher $K d V$ s on the set of solutions of the stationary problem one has the formula, in terms of the gap boundaries,

$$
\begin{aligned}
& R(E)=\prod_{i=1}^{2 n+1}\left(E-E_{i}\right), \\
& Q_{i}=2^{2 k+3}(-1)^{n+k} \sum_{k_{1}+k_{2}+\cdots k_{2 n+1}=n+i+2} \beta_{k_{i}} \cdots \beta_{k_{2 n+1}} E_{1}^{k_{1}} \cdots E_{2 n+1}^{k_{2 n+1}},
\end{aligned}
$$

where

$$
\beta_{n}=\frac{m(m-1) \cdots(m-n+1)}{n !}, m=\frac{1}{2}
$$

This concludes our survey of the periodic theory of the $K d V$ and Schrödinger equations. A complete overview of this theory, and of the relevant literature, may be found in [4]. We remark that in his paper [3] in 1974, P. Lax showed (at the same time as the authors) that smooth real periodic solutions of the stationary higher $K d V$ s are finitegap potentials. His proof was non-constructive, and did not lead to a construction of the integrals, nor to formulae for the gap boundaries (the number of gaps was not determined either). Later, in [5], Lax developed his method and gave the construction of integrals outlined above; this was also done by Gel'fand and Dikii [6]. Furthermore, the work of McKean-van Moerbeke [9] appeared in 1975; this contains some of the present author's results (see [2]), in particular, the complex Jacobi varieties associated with the Riemann surface were obtained. One must also call attention to the little-known work of Ahiezer [13], where finite-gap potentials were first constructed by the use of ideas from Riemann-surface theory.

6. Let us now turn to generalizations. Firstly, the translation of these methods to the so-called Toda lattice, the nonlinear Schrödinger equation, and others where two-sheeted Riemann surfaces occur, presents no difficulty (as noted in the survey [4], in preprints of Tanaka and Date, Kac and van Moerbeke, Flaschka and McLaughlin, in the work of Its, etc.). We shall not discuss these further. Considerably more complicated are problems in which many-sheeted Riemann surfaces occur. Such arise in the matrix equations of Zaharov and Sabat [10]; these are of the form $\dot{L}=[A, L]$, where 


$$
\begin{aligned}
L=\frac{\partial}{\partial x}+Q^{(1)}, A= & \frac{\partial}{\partial x}+Q^{(2)}, Q_{i i}^{(1)}=Q_{i i}^{(2)} \\
& =0, \quad i=1, \cdots, n,
\end{aligned}
$$

$Q^{(\alpha)}$ being $n \times n$ matrices.

A special case of physical interest is the $n$-wave system of Zaharov and Manakov. For $n=3$, these equations have also been studied by Kaup; they are:

$$
\frac{\partial u_{\alpha}}{\partial t}+v_{\alpha} \frac{\partial u_{\alpha}}{\partial x}=i u_{\gamma} u_{\delta} \quad \alpha, \gamma, \delta=1,2,3, \quad \alpha \neq \gamma \neq \delta,
$$

or

$$
\frac{\partial u_{\alpha}}{\partial t}+v_{\alpha} \frac{\partial u_{\alpha}}{\partial x}= \begin{cases}i q u_{2} u_{3}, & \alpha=1 \\ i q^{*} u_{1} u_{3}^{*}, & \alpha=2 \\ i q^{*} u_{1} u_{2}{ }^{+}, & \alpha=3\end{cases}
$$

Dubrovin carried out a study of the periodic problem for these equations, introducing an analog of the "higher $K d V \mathrm{~s}$ " and of "finite-gap operators." Here one has a more complicated version of all the abovementioned constructions and formulae. There is a natural definition of the translation matrix $\hat{T}$, of the "Bloch functions" $\psi_{\alpha}\left(x, x_{0}, E\right)$ $(\alpha=1, \cdots, n)$ which are meromorphic on an $n$-sheeted Riemann surface $R$, and one finds a class of "finite-gap" operators $L$, for which the surface $R$ is algebraic. However, the analytic properties of $\psi$ are complicated. We refer to the survey [4], where a portion of this theory is developed. Further results will appear in Funk. Anal. Priloz.

Now we turn in some detail to a new paper of I. M. Kričever, "Algebro-geometric construction of the Zaharov-Sabat equations, and their periodic solutions," which will appear in the Doklady Akad Nauk SSSR, and is not described in [4]. Amongst these equations, in particular, there is the "two-dimensional $K d V$ equation," first derived by B. B. Kadomtsev et al. (referenced in [10]) in connection with the problem of the stability of the $K d V$ solutions under transverse perturbations:

$$
\begin{aligned}
3 \frac{\partial^{2} u}{\partial y^{2}}+ & \frac{\partial}{\partial x}\left(6 u \frac{\partial u}{\partial x}+\frac{\partial^{3} u}{\partial x^{3}}\right. \\
& \left.+\lambda \frac{\partial u}{\partial x^{3}}+4 \frac{\partial u}{\partial t}\right)=0 .
\end{aligned}
$$

Following Zaharov and Sabat [10], these equations can be written in the form 


$$
-\frac{\partial L}{\partial t}+\frac{\partial A}{\partial y}=[A, L] \text {. }
$$

The methods of [10] allow one to find solutions decaying for $|x| \rightarrow \infty$. The new idea of Kričever is this. Consider an arbitrary Riemann surface $R$. Fix points $P_{o}, Q_{1}, \cdots, Q_{n}$ on $R, n$ being the genus of $R$. Let $z$ be a local coordinate near $P_{0}$ (which corresponds to $z=0$ ), and let $k=1 / z$. Following Ahiezer [13], one may always construct a function $\psi(x, y, t, P)$ depending on parameters $x, y, t$ and on $P \in R$, such that:

1. The poles of $\psi$ are simple, independent of $x, y, t$, and are located at the $Q_{j}$;

2. $\psi \sim \exp \left\{k x+\alpha(k) y+\beta_{m}(k) t\right\}$ as $k \rightarrow \infty$;

3. $\psi$ is meromorphic on $R$ away from $P_{0}$.

Such a function $\psi$ can be given explicitly, thanks to an idea of Its (see [4], Appendix 3). Above, $\alpha(k)$ and $\beta_{m}(k)$ are constant-coefficient polynomials of degree $\ell$ and $m$, respectively. The zeroes $Q_{j}(x, y, t)$ of $\psi(j=1, \cdots, n)$ turn out to be convenient variables in our subsequent considerations.

Proposition 1. There are uniquely determined differential operators of order $\ell$ and $m$ and coeffcients depending on $(x, y, t)$, such that

$$
L_{\iota} \psi=\frac{\partial \psi}{\partial y_{i}}, L_{m} \psi=\frac{\partial \psi}{\partial t}
$$

where

$$
\begin{aligned}
& L_{\ell}=\sum_{i=0}^{\ell} a_{i} \frac{d^{i}}{d x^{i}} \cdot L_{m}=\sum_{i=0}^{m} b_{i} \frac{d^{i}}{d x^{i}}, \\
& a_{\ell}=\alpha=\text { const, } b_{m}=\beta=\text { const. }
\end{aligned}
$$

Proposition 2. The integrability conditions

$$
\frac{\partial^{2} \psi}{\partial y \partial t}=\frac{\partial^{2} \psi}{\partial t \partial y} \text { or } \frac{\partial L_{m}}{\partial y}-\frac{\partial L_{\ell}}{\partial t}=\left[L_{\beta}, L_{m}\right]
$$

are satisfied.

Proposition 3. This construction yields the Zaharov-S̆abat equations [10] for the coefficients of the operators $L_{\rho} L_{m}$. In particular, if $\alpha_{\ell}=k^{2}+c, \beta_{m}=k^{3}+(3 / 2) c k$, one obtains the Kadomtsev-Petriašvili equation in which

$$
L_{2}=\frac{d^{2}}{d x^{2}}+u(x), A=L_{3}=\frac{d^{3}}{d x^{3}}+\frac{3}{2} u \frac{d}{d x}+v
$$


Proposition 4. Let $\psi$ be written in the form

$$
\psi=\psi_{0}(x, t, P) \exp (H y)
$$

where $H(P)$ is meromorphic on $R$, independent of $(x, y, t)$, and has principal part $H \sim \alpha_{\lambda}(k)$ near $P_{0}$. Then

$$
L_{\ell} \psi_{0}=H \psi_{0}, \frac{\partial L_{\ell}}{\partial y} \equiv \frac{\partial L_{m}}{\partial y} \equiv 0, \quad \frac{\partial L_{\ell}}{\partial t}=\left[L_{m}, L_{\ell}\right]
$$

For example, if $\alpha_{\ell}(k)=k^{2}$, then $L_{\ell}$ is the Schrödinger operator, $\psi_{0}$ is the Bloch function, $P_{0}$ may be taken to be a ramification point and $R$ is a two-sheeted (hyperelliptic) Riemann surface. Another example: interchange the roles of $t$ and $y$. Let $\psi=\psi_{0}(x, y, p) \exp (H t)$, with the principal part of $H$ near $P_{0}$ being of the form $k^{3}$. We obtain "finite-gap" solutions of the equations of the nonlinear string (Translator's note: Boussinesq equation),

$$
\partial L_{3} / \partial y=\left[L_{2}, L_{3}\right]
$$

The Riemann surface may be three-sheeted.

These solutions of the Zaharov-Sabat equations are conditionally periodic in $x, y, t$. They are polynomials in $d^{r} / d x^{r} \xi_{s}(x, y, t), r \geqq 0$, arising as coefficients in the expansion about $P_{0}$ of the function

$$
\psi(x, y, t) \exp \left(-k x-\alpha_{\lambda}(k) y-\beta_{m}(k) t\right)=1+\sum_{i=1}^{\infty} \xi_{i}(x, y, t) z^{i}
$$

Explicit formulas may be obtained from the following result.

Proposition 5. The coefficients $\chi_{s}(x, y, t)$ of the series

$$
\frac{\partial}{\partial x} \ln \left(1+\sum_{i=1}^{\infty} \xi_{i}(x, y, t) z^{i}\right)=\sum_{s=1}^{\infty} \chi_{s}(x, y, t) z^{s}
$$

are given by the formula

$$
\begin{aligned}
\frac{\partial}{\partial x} & \sum\left(\prod_{k=1}^{s} \frac{1}{\left(k \alpha_{k}\right) !} \frac{\partial^{\alpha_{1}+\cdots+\alpha_{s}}}{\partial_{\eta_{1}}^{\alpha_{1}} \cdots \partial_{\eta_{s}}^{\alpha_{s}}} \ln \theta(\vec{U} x\right. \\
& \left.\left.+\vec{V} y+\vec{W} t+\vec{Z}+\sum_{k=1}^{s} \vec{U}^{(k)} \eta_{k}\right)\left.\right|_{\eta_{k}=0}\right) .
\end{aligned}
$$

Here $\vec{U}, \vec{v}, \vec{W}, \vec{U}^{k)}$ are vectors of the $(1 / 2 \pi i) b$-periods of differentials with singularity at $P_{0}: d(1 / z), d\left(\alpha_{\alpha}(1 / z)\right), d\left(\beta_{m}(1 / z)\right), k !\left(d z / z^{k+1}\right)$; the vector $\vec{Z}$ corresponds to the divisor $Q_{1}, \cdots, Q_{n}$ under the Abel transform. The summation is extended over all $\alpha_{1}, \cdots, \alpha_{s}$ satisfying $\sum_{k=1}^{s} k \alpha_{k}=s$. 
Since $\vec{U}=-\vec{U}^{(1)}$, we have $\partial / \partial \eta_{1}=-\partial / \partial x$, and find for the solution of the Kadomtsev-Petriašvili equation:

$$
\begin{aligned}
u(x, y, t) & =c-2 \frac{\partial}{\partial x} \xi_{1}(x, y, t) \\
& =c+2 \frac{\partial^{2}}{\partial x^{2}} \ln \theta(\vec{U} x+\vec{V} y+\vec{W} t+\vec{Z}) .
\end{aligned}
$$

These solutons have physical relevance provided that $\vec{V}$ is small compared to $\vec{U}$. On a two-sheeted surface, this means that $P_{0}$ is close to the ramification point, since $\vec{V}=0$ when the two coincide.

This framework may well lead to the solution of an interesting mathematical problem: to classify all commutative algebras of differential operators in the variable $x$ (see Kričever's Doklady article). [Added in proof. Remarkable results in this problem were obtained firstly by $\mathrm{J}$. L. Burchnall, T. W. Chaundy, and H. E. Baker (see appendix in the survey of Kričever, UMN XXXII N6 (1977), 183-208-Soviet Mathematical Surveys).]

\section{REFERENCES}

1. S. P. Novikov, The Periodic Problem for the Korteweg-deVries Equation, Funk. Anal. Prilož. 8 (1974), 54-66.

2. B. A. Dubrovin, S. P. Novikov, Periodic and Quasiperiodic Analogs of the Multisoliton Solutions of the KdV Equation, Zurnal Eksp. i. Teor. Fiz. 67 (1974), 2131-2143.

3. P. D. Lax, Periodic Solutions of the KdV Equations, Lectures in Applied Mathematics, V. 15, A. C. Newell, ed., AMS, Providence, R.I., 1974.

4. B. A. Dubrovin, V. B. Matveev, S. P. Novikov, Nonlinear Korteweg-deVries-like Equations, Finite-gap Operators, and Abelian Varieties, Uspehi Mat. Nauk 31 (1976), 55-136.

5. P. D. Lax, Periodic Solutions of the KdV Equation, Comm. Pure Appl. Math. 28 (1975), 141-188.

6. I. M. Gel'fand, L. A. Dikiǐ, Asymptotics of the Resolvent of Sturm-Liouville Equations and the Algebra of the KdV Equation, Uspehi Mat. Nauk 30 (1975), 67-100.

7. O. I. Bogojavlenskiǐ, S. P. Novikov, On the Hamiltonian Formalisms in Stationary and Nonstationary Problems, Funkts. Anal. Prilož. 10, No. 1 (1976), 9-13.

8. O. I. Bogojavlenskii, On the Integrals of the Higher KdV Equations and the Eigenvalues of Hill's Operator, Funkts. Anal. Prilož. 10, No. 2 (1976), 9-12.

91. H. P. McKean, P. van Moerbeke, The Spectrum of Hill's Equation, Invent. Math. 30 (1975), 217-274.

10. V. E. Zaharov, A. B. Sabat, Scheme for Integrating Equations of Mathematical Physics by the Inverse Scattering Method, Funkts. Anal. Prilož. 8, No. 3 (1974), 43-54.

11. B. A. Dubrovin, Periodic Problem for the KdV Equation in the Class of Finite-Gap Potentials, Funkts. Anal. Prilož. 9, No. 3 (1975), 41-52.

12. A. R. Its, V. B. Matveev, Schrödinger Operators with Finite-Gap Spectrum and NSoliton Solutions of the KdV Equation, Teoret. Mat. Fizika 23 (1975), 51-68.

13. N. I. Akhiezer, Continuous Analogues of Orthogonal Polynomials on a system of Intervals, Dokl. Akad. Nauk USSR 141 (1961), 263-266.

Landau Institute of Theoretical Physics, Moscow, U.S.S.R. 
